\title{
Scintigraphic Evaluation of Takotsubo Cardiomyopathy
}

\author{
Omosalewa O. Lalude*, ${ }^{* 1}$ Mell F. Gutarra ${ }^{2}$ and Aamer Abbas ${ }^{1}$
}

${ }^{I}$ Texas Tech University Health Sciences Center, Cardiology Division-Department of Internal Medicine, 4800 Alberta Avenue, El Paso, TX 79905, USA; ${ }^{2}$ Texas Tech University Health Sciences Center, Department of Internal Medicine, 4800 Alberta Avenue, El Paso, TX 79905, USA

\begin{abstract}
The Takotsubo Cardiomyopathy (TC) also called broken heart syndrome, or stress-induced cardiomyopathy is characterized by the transient ballooning of the apex during systole as result of apical dyskinesia in conjunction with a compensatory hyperkinesia of the base of the left ventricle; in the absence of significant epicardial coronary artery disease. We report a case of TC with the characteristic findings of apical dyskinesia and perfusion abnormalities on gated SPECT which was diagnosed in retrospect after coronary angiography and left ventriculography. This is followed by a brief review of the scintigraphic techniques that have been used in the evaluation of TC. As TC accounts for at least $1-2 \%$ of presentations of acute coronary syndromes, increased awareness on the part of interpreting physicians is required for earlier diagnosis in the subset of patients referred for myocardial perfusion imaging.
\end{abstract}

Keywords: Takotsubo, cardiomyopathy, SPECT, PET.

\section{INTRODUCTION}

The Takotsubo Cardiomyopathy (TC) also called broken heart syndrome, or stress-induced cardiomyopathy is characterized by the transient ballooning of the apex during systole as result of apical dyskinesia in conjunction with a compensatory hyperkinesia of the base of the left ventricle; in the absence of significant epicardial coronary artery disease (CAD). It usually occurs after exposure to a stressful event and was first described by Dote and colleagues in 1991 [1]. The name TC is derived from the similarity of the left ventricular morphology in systole to a trap used in Japan to catch octopus.

TC can present with symptoms similar to CAD (Chest pain, shortness of breath, etc) in the presence of ECG changes and mild elevation of cardiac enzymes. It seems to be more frequent in women of post menopausal age [2] but the exact prevalence is unknown. Akashi and colleagues found that the prevalence of $\mathrm{TC}$ in their institution was around 2\% [3] similar to what Bybee and co-workers reported [4]. New cases of TC are reported every year around the world and nuclear medicine techniques have been used in evaluation of several of these cases. We present the following case in which gated SPECT contributed significantly to diagnosis followed by a brief review of the different scintigraphic techniques that have been used in the evaluation of TC.

\section{CASE REPORT}

A 77 year old Latino American woman with past medical history of hypertension, dyslipidemia, anemia, paroxysmal

\footnotetext{
*Address correspondence to this author at the Texas Tech University Health Sciences Center, Cardiology Division-Department of Internal Medicine, 4800 Alberta Avenue, El Paso, TX 79905, USA; Tel: 915-545-6626; Ext: 258; Fax: 915-545-6634; E-mail: Omosalewa.Lalude@ttuhsc.edu
}

atrial fibrillation and chronic kidney disease on peritoneal dialysis was admitted to the telemetry unit for chest pain associated with shortness of breath, after an episode of near syncope. On physical exam, the patient was noted to be pale with a heart rate of 102/minute and a blood pressure of $149 / 86 \mathrm{~mm} \mathrm{Hg}$. There were no significant findings on the cardiovascular exam and the lungs were clear to auscultation. The rest of the physical exam was unremarkable.

The initial ECG (Fig. 1) on admission showed sinus rhythm at a rate of $96 / \mathrm{min}$ with $\mathrm{T}$-wave inversion in leads I, aVL, V4-6, poor R wave progression, pseudo inferior infarct pattern, left axis deviation and QT prolongation (QTc interval $517 \mathrm{msec}$ ). The QT was slightly prolonged compared to prior ECGs. The laboratory tests revealed a hemoglobin of $9 \mathrm{~g} / \mathrm{dL}$ (normal $12-15 \mathrm{~g} / \mathrm{dL}$ ) and maximum Troponin I of 0.22 $\mathrm{ng} / \mathrm{mL}$ (normal value $<0.04 \mathrm{ng} / \mathrm{mL}$ ). The minimal Troponin elevation was attributed to anemia and renal failure. The patient was transfused with packed red blood cells and managed medically. She had no recurrence of chest pain and an out-patient stress test was requested.

An adenosine Tc-99m Tetrofosmin SPECT study was performed 3 days after initial presentation. Although a twoday study was planned, she did not return for the rest study until 5 days later. The stress test was interpreted as a large partially reversible defect consistent with myocardial infarction with peri-infarct ischemia in the left anterior descending artery territory (Fig. 2).

Twelve days after the initial presentation, she returned to the hospital for palpitations, shortness of breath associated with dizziness and anxiety. The ECG (Fig. 3) this time, showed sinus rhythm at a rate of $75 / \mathrm{min}$, tall inferior $\mathrm{R}$ waves, a premature ventricular contraction, worsening of the QT prolongation (QTc interval $638 \mathrm{msec}$ ) and diffuse giant $\mathrm{T}$-wave inversions typical of the last phase of TC. 


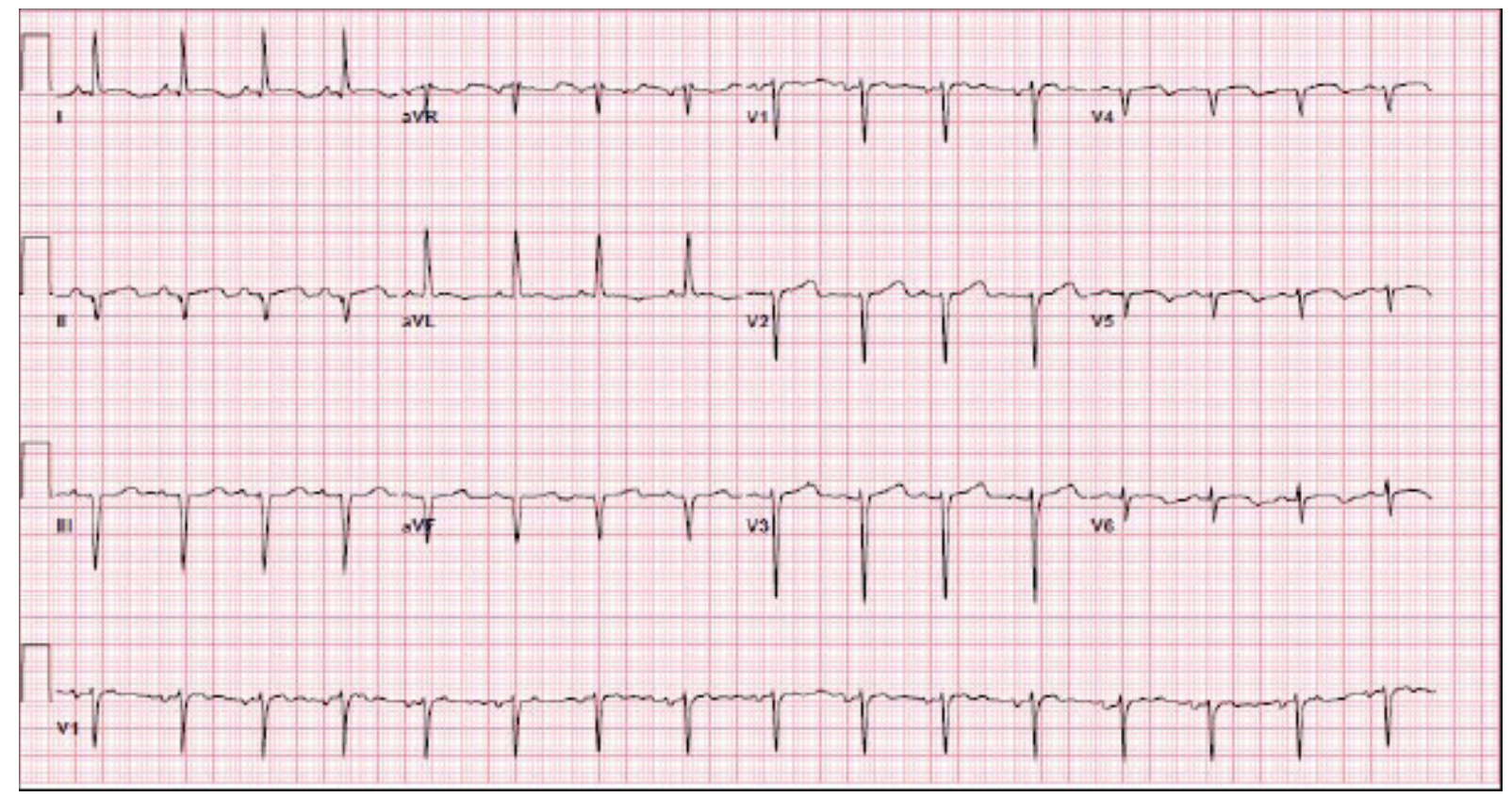

Fig. (1). Electrocardiogram on initial presentation showing sinus rhythm at a rate of 96/min with T-wave inversion in leads I, aVL, V4-6, poor R wave progression, pseudo inferior infarct pattern, left axis deviation and QT prolongation (QTc interval $517 \mathrm{msec}$ ).
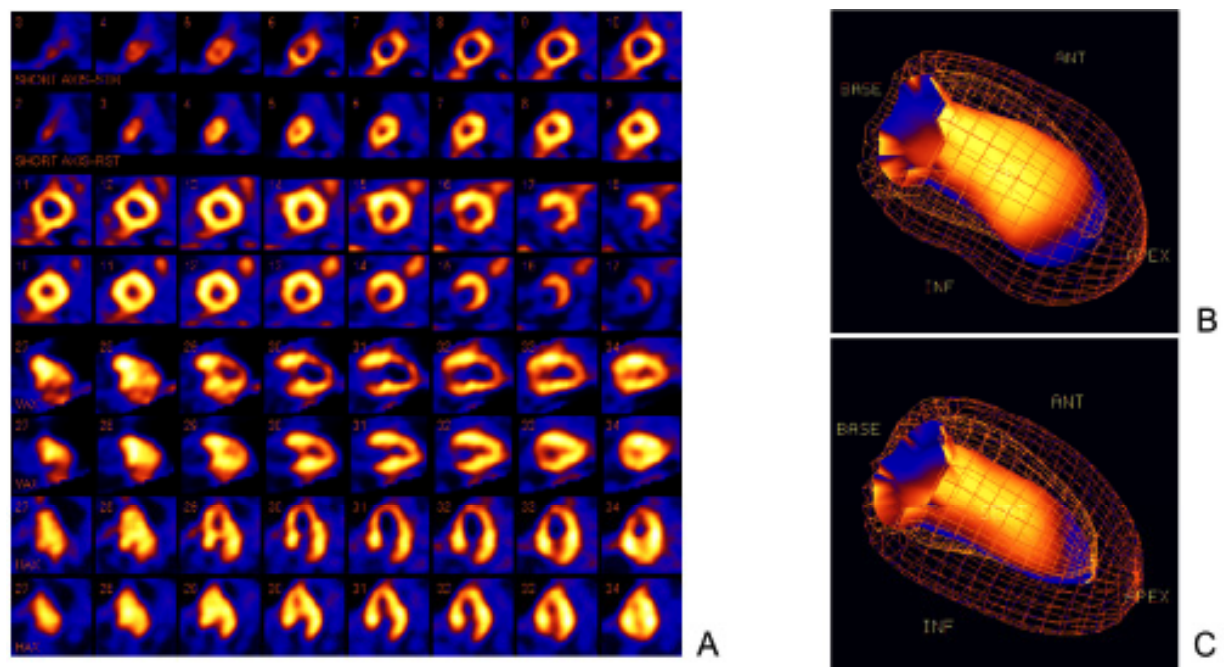

Fig. (2). Tetrofosmin myocardial perfusion gated images showing a large partially reversible perfusion defect (A) associated with severe left ventricular dilatation, wall thinning and dyskinesia of the apical segments on the stress study, ejection fraction (EF) $40 \%$ (B) and severe hypokinesis the apical segments on the rest study, EF $49 \%$ (C).

Cardiac enzymes were again only minimally elevated: Troponin I 0.65 (normal $0-0.04 \mathrm{ng} / \mathrm{mL}$ ), total CK $30 \mathrm{IU} / \mathrm{L}$ (normal 32-152 IU/L) and CKMB $5.9 \mathrm{ng} / \mathrm{mL}$ (normal 0.6$6.3 \mathrm{ng} / \mathrm{mL})$. However, given the recent hospitalization and abnormal stress test, left heart catheterization/coronary angiography was performed (Fig. 4). The left ventricular systolic function was normal with ejection fraction (EF) of $65 \%$ and there was complete resolution of the wall motion abnormalities previously noted on the SPECT study. The coronaries were normal except for a $70 \%$ stenosis of a small caliber second diagonal branch of the left anterior descending artery which did not explain the findings on the perfusion study. The clinical, electrocardiographic, scintigraphic and angiographic findings were thought to be consistent with Takotsubo cardiomyopathy (TC).

\section{DISCUSSION}

Classic TC is a reversible myocardial dysfunction with a transitory perfusion defect [5]. Differentiating TC from an acute coronary syndrome remains a diagnostic challenge. TC can be evaluated via several nuclear imaging techniques. Gated SPECT with Tc-99m Tetrosfosmin/Sestamibi has been used successfully and the classic finding is a fixed perfusion defect that cuts across several coronary vascular territories with apical ballooning and dyskinesia. In this case, the partially reversible defect is explained by the delay in obtaining rest images following stress imaging. In keeping with other published studies however, myocardial perfusion was not completely absent even on the stress study [6]. In fact, some reports have noted that myocardial perfusion may be completely normal despite significant wall motion abnor- 


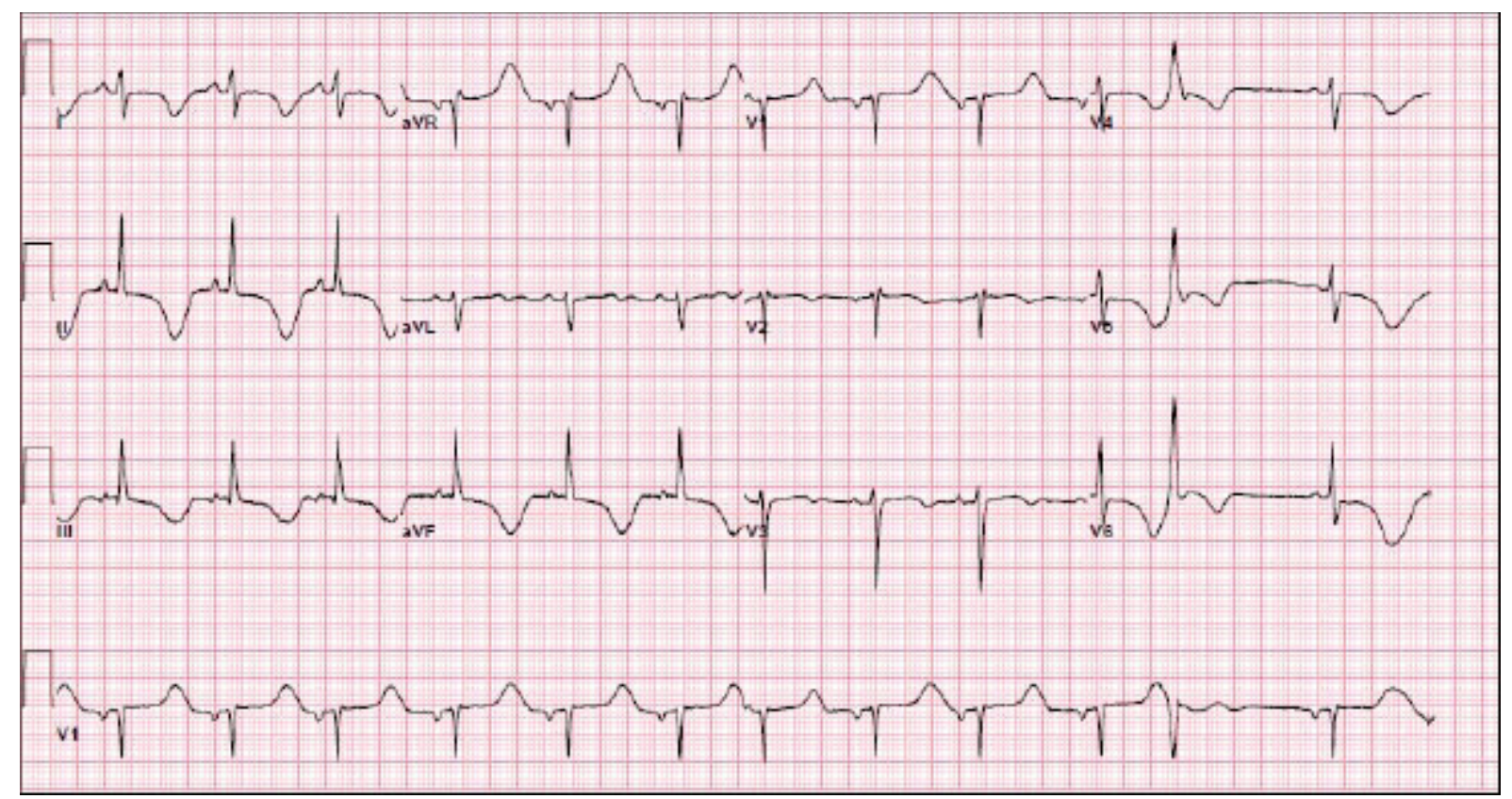

Fig. (3). Electrocardiogram performed 12 days after initial presentation showing sinus rhythm at a rate of $75 / \mathrm{min}$, tall inferior $\mathrm{R}$ waves, a premature ventricular contraction, worsening of the QT prolongation (QTc interval $638 \mathrm{msec}$ ) and diffuse giant T-wave inversions typical of the last phase of TC.
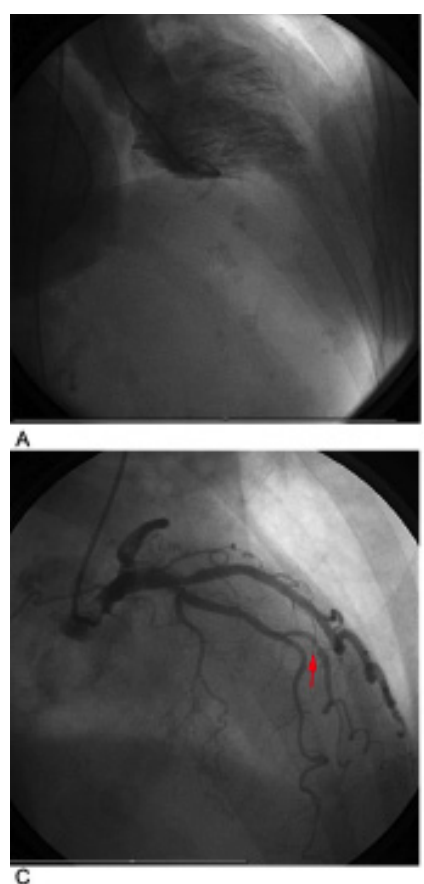
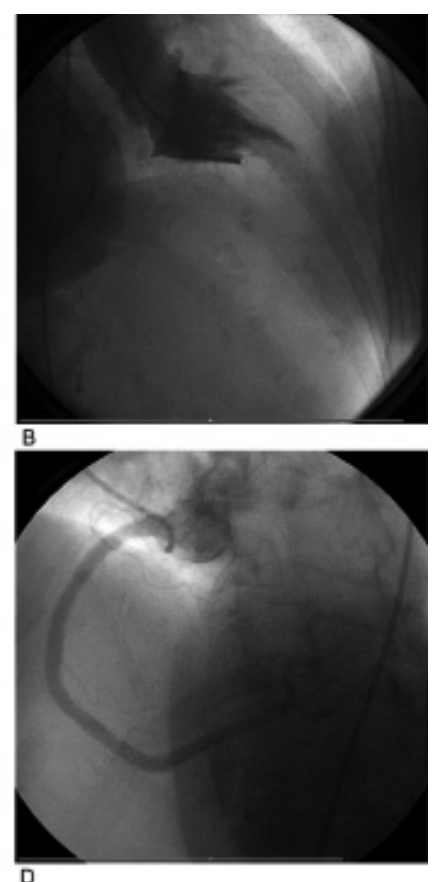

Fig. (4). Right anterior oblique view of the left ventricle in end-diastole (A) and end-systole (B) showing normal systolic function EF $65 \%$. Antero-posterior cranial view of the left coronary system (C), showing a focal $70 \%$ stenosis in the proximal segment of the second diagonal branch (red arrow). No significant disease is seen in the left anterior descending and left circumflex coronary arteries. Antero-posterior cranial view of the dominant right coronary artery (D) shows no significant disease.

malities and the presence of symptoms [7, 8]. Although the perfusion abnormality in the apex can be partially explained by a partial volume effect involving the non-thickening apical segments $[9,10]$, many studies have demonstrated that microvascular dysfunction may be responsible for a true perfusion defect $[6,11,12]$. Another tracer Tc-99mPYP, which has traditionally been used in the diagnosis and quantification of myocardial infarction, has also been used in the evaluation of TC. It binds to calcium hydroxyapatite crystals in necrotic myocardium but has been shown to be taken up by viable but severely damaged or ischemic myocardial cells as in TC [6].

In addition to microvascular dysfunction, metabolic abnormalities have been described. Reduction in fatty acid metabolism occurs under conditions of ischemia and has been documented through SPECT imaging with I-123 BMIPP [6, 
12]. On PET imaging, reduced perfusion with N-13 ammonia [13] and decreased metabolism with F-18 FDG imaging has also been described $[7,13]$. The radio-iodinated analog for norepinephrine I-123 MIBG has also been used to demonstrate a suppression of myocardial sympathetic nerve function in response to myocardial ischemia in TC. These findings of transient decreased perfusion with reduction in metabolism and sympathetic nerve function are characteristic of myocardial stunning. In the sub-acute phase of this case (Day3-8), perfusion defects were seen on the Tc-99m Tetrofosmin myocardial perfusion gated SPECT study. This finding in an elderly female patient with risk factors for CAD led to left heart catheterization. By the time left ventriculography was performed on day 14 , the wall motion abnormalities had completely resolved.

In conclusion, we report a case of TC with the characteristic findings of apical dyskinesia and perfusion abnormalities on gated SPECT which was diagnosed in retrospect after coronary angiography and left ventriculography. As TC accounts for at least $1-2 \%$ of presentations of acute coronary syndromes, increased awareness on the part of interpreting physicians is required for earlier diagnosis in the subset of patients referred for myocardial perfusion imaging. In addition, knowledge of the different scintigraphic imaging modalities and the characteristic findings of TC will enable differentiation of TC from symptomatic coronary artery disease.

\section{REFERENCES}

[1] Dote K, Sato H, Tateishi H, Uchida T, Ishihara M. Myocardial stunning due to simultaneous multivessel coronary spasms: a review of 5 cases. J Cardiol 1991; 21(2): 203-14.
[2] Gianni M, Dentali F, Grandi AM, Sumner G, Hiralal R, Lonn E. Apical ballooning syndrome or takotsubo cardiomyopathy: a systematic review. Eur Heart J 2006; 27(13):1523-29.

[3] Akashi YJ, Nakazawa K, Sakakibara M, Miyake F, Musha H, Sasaka K. 123I-MIBG myocardial scintigraphy in patients with "takotsubo" cardiomyopathy. J Nucl Med 2004; 45(7): 1121-27.

[4] Bybee KA, Prasad A, Barsness GW, et al. Clinical characteristics and thrombolysis in myocardial infarction frame counts in women with transient left ventricular apical ballooning syndrome. Am J Cardiol 2004; 94(3): 343-46.

[5] Alexanderson E, Cruz P, Talayero JA, Damas F, Zeron J, Meave A. Transient perfusion and motion abnormalities in takotsubo cardiomyopathy. J Nucl Cardiol 2007; 14(1):129-33.

[6] Ito K, Sugihara H, Kinoshita N, Azuma A, Matsubara H. Assessment of Takotsubo cardiomyopathy (transient left ventricular apical ballooning) using 99mTc-tetrofosmin, 123I-BMIPP, 123I-MIBG and 99mTc-PYP myocardial SPECT. Ann Nucl Med 2005; 19(6): 435-45.

[7] Cimarelli S, Imperiale A, Ben-Sellem D, et al. Nuclear medicine imaging of takotsubo cardiomyopathy: typical form and midventricular ballooning syndrome. J Nucl Cardiol 2008; 15(1):137-41.

[8] Dorfman T, Aqel R, Allred J, Woodham R, Iskandrian AE. Takotsubo cardiomyopathy induced by treadmill exercise testing: an insight into the pathophysiology of transient left ventricular apical (or midventricular) ballooning in the absence of obstructive coronary artery disease. J Am Coll Cardiol 2007; 49(11): 1223-25.

[9] Dorfman TA, Iskandrian AE. Takotsubo cardiomyopathy: state-ofthe-art review. J Nucl Cardiol 2009; 16(1): 122-34.

[10] Davis M, Hardebeck C. Reverse Takotsubo syndrome diagnosed with Tc-99m SPECT perfusion study. J Nucl Cardiol 2009; 16(6): 999-1002.

[11] Kume T, Akasaka T, Kawamoto T, et al. Assessment of coronary microcirculation in patients with takotsubo-like left ventricular dysfunction. Circ J 2005; 69(8): 934-39.

[12] Sato A, Aonuma K, Nozato T, et al. Stunned myocardium in transient left ventricular apical ballooning: a serial study of dual I-123 BMIPP and Tl-201 SPECT. J Nucl Cardiol 2008; 15(5): 671-79.

[13] Bybee KA, Murphy J, Prasad A, et al. Acute impairment of regional myocardial glucose uptake in the apical ballooning (takotsubo) syndrome. J Nucl Cardiol 2006; 13(2): 244-50.

(C) Lalude et al.; Licensee Bentham Open.

This is an open access article licensed under the terms of the Creative Commons Attribution Non-Commercial License (http: //creativecommons.org/licenses/by$\mathrm{nc} / 3.0 /$ ) which permits unrestricted, non-commercial use, distribution and reproduction in any medium, provided the work is properly cited. 